SLAC-PUB-6196

SCIPP-93/08

SMU HEP 93-06

May 1993

$\mathrm{T} / \mathrm{E}$

\title{
Constraints on CP violation in the Higgs sector from the $\rho$ parameter ${ }^{\star}$
}

\author{
Alex Pomarol \\ Santa Cruz Institute for Particle Physics \\ University of California, Santa Cruz, CA 95064 \\ and \\ Roberto Vega \\ Stanford Linear Accelerator Center \\ Stanford University, California 94309 \\ and \\ Department of Physics, Southern Methodist University \\ Dallas, Texas 75275-0175
}

\begin{abstract}
We discuss the relation between the CP symmetry and the custodial $S U(2)$ symmetry in the Higgs sector. In particular, we show that $\mathrm{CP}$ violation in the Higgs-gauge sector is allowed only if the custodial $S U(2)$ symmetry is broken. We exploit these facts to constrain CP violation using the experimental bounds on $\rho-1$. $\mathrm{CP}$ nonconservation in the Higgs-fermion interactions can also be constrained in a similar way although a possible exception is pointed out.
\end{abstract}

Submitted to Physical Review D.

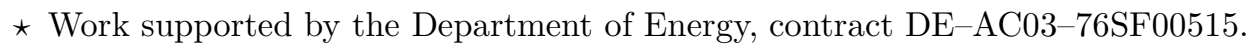




\section{Introduction}

In the standard model (SM) of the weak interactions the origin of CP nonconservation is intimately related to the mechanism of electroweak symmetry breaking (ESB). Progress in the understanding of one of these phenomena will most probably lead to progress in the understanding of the other.

CP nonconservation in the SM arises from the phases appearing in the KobayashiMaskawa matrix ${ }^{1}$. This suffices to explain the $\mathrm{CP}$ violation observed in the neutral kaon system. However, unless only one Higgs doublet triggers ESB, it seems natural for CP nonconservation to also occur in the Higgs interactions ${ }^{2}$. This source of $\mathrm{CP}$ violation can manifest itself through virtual effects or through direct production of Higgs bosons. In the first case, one looks for one-loop Higgs contributions to CP-violating experimental observables such as the electric dipole moment of the neutron $^{2-4}$ and electron ${ }^{5}$, or asymmetries in the top quark production or decay ${ }^{6}$. Such one-loop contributions are generally small and decrease with increasing Higgs masses. For Higgs masses of the order of a hundred GeV maximal $\mathrm{CP}$ violation in the Higgs sector is consistent with present experimental data. In the second case, one tries to detect $\mathrm{CP}$ violation in the production mechanisms of the Higgs bosons or their decays $^{7-9}$. For example, evidence of CP violation in the production of two Higgs, $H_{1}$ and $H_{2}$, in $e^{+} e^{-}$colliders can be obtained by observing the processes ${ }^{7}$

$$
\begin{aligned}
e^{+} e^{-} \rightarrow Z^{*} & \rightarrow Z H_{1}, \\
& \rightarrow Z H_{2}, \\
e^{+} e^{-} \rightarrow Z^{*} & \rightarrow H_{1} H_{2} .
\end{aligned}
$$

Another possibility would be to use polarized photons produced by back-scattering laser beams at a $\mathrm{TeV}$ scale $e^{+} e^{-}$collider $^{8}$. In this case, large Higgs production asymmetries in $\gamma \gamma$ collisions would provide evidence of $\mathrm{CP}$ violation. 
In this paper we propose a new way to constrain CP violation in the Higgs sector. The idea is as follows. We know that experimentally $\rho \equiv m_{W}^{2} /\left(m_{Z}^{2} \cos ^{2} \theta_{W}\right) \simeq$ 1. This can be understood as a consequence of an approximate global $S U(2)$ symmetry of the lagrangian, called in the literature a custodial symmetry ${ }^{10}$. We will show that when we have CP violation in the Higgs sector, such a custodial $S U(2)$ symmetry cannot be defined in the Higgs potential. ${ }^{\# 1}$ Thus, if we insist in having $\mathrm{CP}$ violation, radiative corrections to $\rho$ will be unavoidable. From the experimental bounds on $\Delta \rho \equiv \rho-1$, we will be able to constrain the $\mathrm{CP}$ violation in the Higgs sector.

In section 2 , we analyze the scalar contributions to the $\rho$ parameter in a CPviolating two Higgs doublet model. The custodial symmetry is defined in section 3 where we prove our assertion that a custodial-invariant Higgs potential is always $\mathrm{CP}$ conserving. In section 4 we use the results of section 2 and 3 in an attempt to place bounds on CP violation in the Higgs sector. Finally, section 5 is devoted to the conclusions.

\section{Contribution to the $\rho$ parameter from a Higgs sector with maximal CP violation}

$\mathrm{CP}$ violation can occur in the Higgs sector in models with several scalar multiplets. Models with only Higgs doublets are particularly interesting because at tree level $\rho=1$ is insured in a natural way. This is true whether or not we impose a custodial symmetry on the scalar potential. Higher representations, on the other hand, require a fine-tuning of the vacuum expectation values (VEVs) of the neutral scalars in order that $\rho \simeq 1$. $^{\# 2}$ In such models the $\rho$ parameter is arbitrary and

\#1 This was previously pointed out by S. Weinberg in Ref. [2].

\#2 There are some exotic representations, such as a 7-plet of hypercharge $Y= \pm 4$, for which $\rho=1$ at tree level independently of the VEVs of the scalars. 
radiative corrections are not calculable.

In this paper we will only work within the two Higgs doublet model (THDM). Our results, however, can be generalized to most of the multi-scalar models. The most general $S U(2)_{L} \times U(1)_{Y}$ gauge invariant two Higgs doublet potential is

$$
\begin{aligned}
V\left(\Phi_{1}, \Phi_{2}\right) & =m_{1}^{2} \Phi_{1}^{\dagger} \Phi_{1}+m_{2}^{2} \Phi_{2}^{\dagger} \Phi_{2}-\left(m_{12}^{2} \Phi_{1}^{\dagger} \Phi_{2}+\text { h.c. }\right) \\
& +\lambda_{1}\left(\Phi_{1}^{\dagger} \Phi_{1}\right)^{2}+\lambda_{2}\left(\Phi_{2}^{\dagger} \Phi_{2}\right)^{2}+\lambda_{3}\left(\Phi_{1}^{\dagger} \Phi_{1}\right)\left(\Phi_{2}^{\dagger} \Phi_{2}\right)+\lambda_{4}\left(\Phi_{1}^{\dagger} \Phi_{2}\right)\left(\Phi_{2}^{\dagger} \Phi_{1}\right) \\
& +\frac{1}{2}\left[\lambda_{5}\left(\Phi_{1}^{\dagger} \Phi_{2}\right)^{2}+\text { h.c. }\right]+\frac{1}{2}\left[\Phi_{1}^{\dagger} \Phi_{2}\left\{\lambda_{6}\left(\Phi_{1}^{\dagger} \Phi_{1}\right)+\lambda_{7}\left(\Phi_{2}^{\dagger} \Phi_{2}\right)\right\}+\text { h.c. }\right] .
\end{aligned}
$$

In general the parameters $m_{12}^{2}, \lambda_{5}, \lambda_{6}$ and $\lambda_{7}$ can be complex and thus give rise to CP violation in the Higgs sector. ${ }^{\# 3}$ Even if all the parameters are real (no explicit $\mathrm{CP}$ violation) it can be shown that there is a region of parameter space where the VEVs of the neutral scalars are

$$
\left\langle\phi_{1}^{0}\right\rangle=v_{1} \quad, \quad\left\langle\phi_{2}^{0}\right\rangle=v_{2} e^{i \xi} \quad \xi \neq n \pi(n \in \mathbf{Z}),
$$

and therefore $\mathrm{CP}$ is spontaneously broken ${ }^{12}$. It is convenient to define a basis $\Phi_{1}^{\prime}$, $\Phi_{2}^{\prime}$ where only one Higgs doublet gets a VEV, i.e.,

$$
\begin{aligned}
& \Phi_{1}^{\prime}=\cos \beta \Phi_{1}+\sin \beta e^{-i \xi} \Phi_{2}=\left(\begin{array}{c}
G^{+} \\
v+\frac{1}{\sqrt{2}}\left(h^{0}+i G^{0}\right)
\end{array}\right), \\
& \Phi_{2}^{\prime}=-\sin \beta \Phi_{1}+\cos \beta e^{-i \xi} \Phi_{2}=\left(\begin{array}{c}
H^{+} \\
\frac{1}{\sqrt{2}}\left(H^{0}+i A^{0}\right)
\end{array}\right),
\end{aligned}
$$

where $v \equiv \sqrt{v_{1}^{2}+v_{2}^{2}}, \tan \beta=v_{2} / v_{1}, G^{+}$and $G^{0}$ are the Goldstone bosons and $H^{+}$ is the charged Higgs. The three neutral Higgs boson mass eigenstates $H_{i=1,3}$ are

\#3 Strictly speaking, the presence of complex scalar self-couplings is a necessary but not sufficient condition for $\mathrm{CP}$ violation ${ }^{11}$. 
mixtures of $h^{0}, H^{0}$ and $A^{0}$ :

$$
\left(\begin{array}{l}
H_{1} \\
H_{2} \\
H_{3}
\end{array}\right)=\boldsymbol{O}\left(\begin{array}{l}
h^{0} \\
H^{0} \\
A^{0}
\end{array}\right),
$$

where $\boldsymbol{O}$ is an orthogonal matrix.

Higgs boson contributions to the $\rho$ parameter have been extensively analyzed in theories with two Higgs doublets without CP violation ${ }^{13-17}$ and also more recently in the CP-violating case ${ }^{18}$. Nevertheless, the connection between the magnitude of such contributions, the custodial symmetry and CP violation in the Higgs sector has not been analyzed.

The loop contributions to $\Delta \rho$ are given by the parameter $T$ defined by ${ }^{19}$

$$
\alpha T \equiv \frac{g^{2}}{m_{W}^{2}}\left[\Pi_{11}(0)-\Pi_{33}(0)\right]
$$

where $\Pi_{a b}\left(q^{2}\right)$ is the coefficient of $g^{\mu \nu}$ in the vacuum polarization tensor and $\alpha=e^{2} /(4 \pi)$. The Higgs contributions to $T$ are not finite. The gauge boson contributions must also be included to obtain a finite result. For this reason, it is convenient to set the SM with one Higgs doublet $\left(H_{r e f}\right)$ as a reference point and study the deviations from this point. In this case, the extra contribution to $T$ in a $\mathrm{CP}$-violating THDM is given by ${ }^{\# 4}$

$$
\begin{aligned}
\alpha T & =-\frac{3 g^{\prime 2}}{6 \pi^{2}} \sum_{i=1}^{3} \frac{\boldsymbol{O}_{i 1}^{2}}{m_{W}^{2}-m_{Z}^{2}} L\left(m_{H_{i}}^{2}, m_{H_{r e f}}^{2}\right) \\
& +\frac{g^{2}}{64 \pi^{2} m_{W}^{2}}\left[\sum_{i=1}^{3}\left(1-\boldsymbol{O}_{i 1}^{2}\right) F\left(m_{H_{i}}^{2}, m_{H^{+}}^{2}\right)-\frac{1}{2} \sum_{\substack{i, j, k=1 \\
i \neq j \neq k}}^{3} \boldsymbol{O}_{i 1}^{2} F\left(m_{H_{j}}^{2}, m_{H_{k}}^{2}\right)\right],
\end{aligned}
$$

\#4 See also Ref. [18]. 
where

$$
L(x, y)=F\left(x, m_{Z}^{2}\right)-F\left(x, m_{W}^{2}\right)+F\left(y, m_{W}^{2}\right)-F\left(y, m_{Z}^{2}\right)
$$

and

$$
F(x, y)=\frac{x+y}{2}-\frac{x y}{x-y} \ln \frac{x}{y}
$$

The first term reflects the breaking of the custodial symmetry which results when the $U(1)_{Y}$ factor is gauged, and arises even when the Higgs potential is custodialinvariant $^{20}$. Such a term grows only logarithmically with the Higgs masses:

$$
\frac{1}{m_{W}^{2}-m_{Z}^{2}} L\left(m_{H_{i}}^{2}, m_{H_{r e f}}^{2}\right) \simeq \ln \frac{m_{H_{i}}^{2}}{m_{H_{r e f}}^{2}} \quad \text { for } m_{H_{i}}, m_{H_{r e f}} \gg m_{Z}
$$

The second term (in brackets) arises, as we will see, only if the Higgs potential does not have a custodial $S U(2)$ symmetry, and gives a contribution that depends quadratically on the Higgs masses. Contrary to what occurs in the CP-conserving THDM, this term does not cancel when the $H^{+}$is degenerate with one neutral Higgs boson ${ }^{13,14}$. From Eq. (7) one can see that this second term vanishes only if there exists a neutral Higgs boson $H_{i}$ such that

$$
m_{H_{i}}=m_{H^{+}} \text {and } \boldsymbol{O}_{i 1}=0
$$

In the case where the $H^{+}$is degenerate with two neutral Higgs such a term also vanishes. It can be easily shown, however, that when this latter condition holds Eq. (11) also holds.

The relation between $\mathrm{CP}$ and the custodial symmetry may be apparent in Eq. (11). It was shown in Ref. [7] that in the limit where $\boldsymbol{O}_{i 1} \rightarrow 0$ (for any $i$ ) CP is conserved by the Higgs-gauge interactions. Consequently, if the contributions to $T$ 
are small then $\mathrm{CP}$ violation is also small. Note that the opposite is not true ${ }^{13-17}$. The relation between the custodial and CP symmetry will become clearer in the next section.

In principle there is no reason to expect that Eq. (11) holds, so the contribution to $T$ for large Higgs masses could be significant. Since $T$ depends on six arbitrary parameters it is difficult to give a full analysis of its value. We will focus on the contribution to $T$ from a maximally CP-violating Higgs sector. This occurs when ${ }^{7}$

$$
O_{11} \simeq O_{21} \simeq O_{31} \simeq \frac{\sqrt{3}}{3}
$$

Maximal CP violation also requires a large splitting between the neutral Higgs masses $^{3,21}$. Since it may be more natural for the Higgs boson mass splitting to be of the order of their masses, i.e.,

$$
\Delta m_{H}^{2} \sim m_{H}^{2}
$$

we will also consider the case where both Eqs. (12) and (13) hold. To give an idea of the orders of magnitude, we have plotted in Fig. 1 the parameter $T$ as a function of the charged Higgs mass for two different sets of neutral Higgs masses. In fig. 1a we have considered a large mass splitting. We have chosen $\left(m_{H_{1}}, m_{H_{2}}, m_{H_{3}}\right)$ $=(60,500,1000) \mathrm{GeV}$ and $(60,1000,1100) \mathrm{GeV}$. In Fig. 1b we have followed the natural condition of Eq. (13) and chosen $m_{H_{1}}=\frac{1}{2} m_{H_{2}}=m_{H_{3}}=\frac{3}{2} m_{H^{+}}$and $m_{H_{1}}=$ $\frac{1}{2} m_{H_{2}}=\frac{3}{2} m_{H^{+}}=2 m_{H_{3}}$. Following Ref. [22], we have taken $m_{H_{r e f}}=m_{t}=m_{Z}$ as the reference point. From Fig. 1a one can see that a large mass splitting results in a large contribution to $T$. This was expected as we noted before. When the Higgs mass splitting is on the order of their masses, the contribution is only relevant for 
a large Higgs mass scale (Fig. 1b). The experimental limits on $T$ (dotted line) have been taken from Ref. [22],

$$
-0.93<T<0.33 .
$$

This experimental bound can be used to rule out a region of parameter space of the Higgs sector. However, since the top quark mass is still unknown there is a large uncertainty in this excluded region (a large negative contribution to $T$ from a CP-violating Higgs sector may be canceled by the positive contribution from the top quark). Nevertheless, it is likely that the top quark will be discovered in the near future. If it were found and its mass measured to an accuracy of $\Delta m_{t} \simeq \pm 5$ $\mathrm{GeV}$, it would be possible ${ }^{23}$, using the "Ultimate LEP" configuration, to improve the experimental uncertainty of $T$ to

$$
\Delta T \simeq \pm 0.1
$$

In this case, if the central value of $T$ remained close to zero (after subtracting the top quark contribution), any custodial-breaking term in the Higgs potential would be tightly constrained. ${ }^{\# 5}$ Of course, we could fine-tune the parameters of the Higgs potential in order to make the contribution to $T$ small without requiring an approximate custodial symmetry. We will not consider such an unnatural possibility.

\section{CP and Custodial Symmetry in the Higgs Potential}

In this section we study the CP symmetry in the limit where the Higgs potential is custodial $S U(2)$ invariant. The custodial symmetry is actually violated

\#5 Actually, due to the first term in Eq. (7), a small experimental value for $T$ would be required even in models with a custodial-invariant scalar potential. 
by the gauge factor $U(1)_{Y}$ and infinite radiative corrections ${ }^{24}$ are induced in a custodial-invariant Higgs potential (unless the gauge group is enlarged -see for example Ref. [25]). Thus, the custodial symmetry can at best be only considered an approximate symmetry.

We need to enlarge the global symmetry of the Higgs potential so that after the ESB there remains a residual $S U(2)$ symmetry. If only three Goldstone bosons are allowed, the symmetry-breaking pattern is

$$
S U(2)_{L} \times S U(2)_{R} \rightarrow S U(2)_{V}
$$

In the THDM there are two possible ways of defining the global $S U(2)_{L} \times S U(2)_{R}$ symmetry $^{26}$ :

Case I: Following the SM case ${ }^{10}$, we define a matrix field

$$
M_{i}=\left(i \tau_{2} \Phi_{i}^{*} \Phi_{i}\right) \equiv\left(\begin{array}{cc}
\phi_{i}^{0 *} & \phi_{i}^{+} \\
-\phi_{i}^{-} & \phi_{i}^{0}
\end{array}\right) \quad i=1,2
$$

which transforms under the $S U(2)_{L} \times S U(2)_{R}$ symmetry as

$$
M_{i} \rightarrow L M_{i} R^{\dagger}
$$

In order that the symmetry is broken down to $S U(2)_{V}$, we need

$$
\left\langle M_{i}\right\rangle=v_{i} \mathbf{1} \quad \Rightarrow \quad\left\langle\phi_{i}^{0}\right\rangle=\left\langle\phi_{i}^{0 *}\right\rangle=v_{i} \in \mathbf{R}
$$

Case II: We define a unique matrix field $M_{21}=\left(i \tau_{2} \Phi_{2}^{*} \Phi_{1}\right)$ which transforms 
under $S U(2)_{L} \times S U(2)_{R}$ as

$$
M_{21} \rightarrow L M_{21} R^{\dagger}
$$

In this case, the symmetry is broken down to $S U(2)_{V}$ when

$$
\left\langle M_{21}\right\rangle \propto \mathbf{1} \Rightarrow\left\langle\phi_{1}^{0}\right\rangle=\left\langle\phi_{2}^{0 *}\right\rangle \in \mathbf{C} .
$$

It can be shown that, in the absence of fermions, transformation (20) is a more restrictive case of transformation (18) . However, since we will be considering the Higgs-fermion interactions we need to consider both cases separately.

We begin with case I and define in the usual way the transformation of the scalar fields under CP:

$$
\begin{aligned}
& \Phi_{1}(\vec{x}, t) \rightarrow \Phi_{1}^{\dagger}(-\vec{x}, t), \\
& \Phi_{2}(\vec{x}, t) \rightarrow \Phi_{2}^{\dagger}(-\vec{x}, t) .
\end{aligned}
$$

Note, that from Eq. (4) and Eq. (19), the above transformation is equivalent to defining $h^{0}$ and $H^{0}$ as CP-even states and $A^{0}$ as a CP-odd state. The transformation (22) can be written in the form

$$
M_{i}(\vec{x}, t) \rightarrow \tau_{2} M_{i}(-\vec{x}, t) \tau_{2} \quad i=1,2 .
$$

Clearly, a Higgs potential that is invariant under (18) (with $L=R$ ) is also invariant under (23). Therefore, a Higgs potential that is invariant under $S U(2)_{V}$ will automatically conserve CP.

For the case II, we define the CP transformation as

$$
\begin{aligned}
& \Phi_{1}(\vec{x}, t) \rightarrow \Phi_{2}^{\dagger}(-\vec{x}, t), \\
& \Phi_{2}(\vec{x}, t) \rightarrow \Phi_{1}^{\dagger}(-\vec{x}, t) .
\end{aligned}
$$

Again this transformation can be written in the form $M_{21}(\vec{x}, t) \rightarrow \tau_{2} M_{21}(-\vec{x}, t) \tau_{2}$. 
It corresponds (using Eq. (4) and Eq. (21)) to defining $h^{0}$ and $A^{0}$ as CP-even states, and $H^{0}$ as CP-odd.

In the appendix we derive the constraints on the most general THDM potential [Eq. (2)] imposed by the $S U(2)_{L} \times S U(2)_{R}$ symmetry. We also show explicitly that $\mathrm{CP}$ is conserved in a THDM with custodial invariance.

The kinetic term for the Higgs bosons is invariant under the $S U(2)_{L} \times S U(2)_{R}$ symmetry only if $g^{\prime}=0$. However, for $g^{\prime} \neq 0$ the transformation $M \rightarrow \tau_{2} M \tau_{2}$ $\left(M=M_{i}\right.$ or $\left.M_{21}\right)$ leaves the kinetic term invariant if the gauge boson $B^{\mu}$ associated with the $U(1)_{Y}$ factor transforms as $B^{\mu} \rightarrow-B^{\mu}$. We conclude then that $\mathrm{CP}$ is conserved by the gauge-Higgs sector if the Higgs potential has a custodial SU(2) symmetry.

Let us now consider the Higgs-fermion interactions. The most general Yukawa sector induces flavor changing neutral currents (FCNC). The usual way of suppressing these is by means of a discrete symmetry ${ }^{27}$ that can be softly broken. CP conservation in the Yukawa interactions requires $h^{0}$ and $H^{0}$ to be CP-even and $A^{0}$ to be CP-odd. Such assignments of CP quantum numbers are compatible with the assignments of case I, but incompatible with those of case II. Therefore, case II allows for the possibility of having an approximate custodial $S U(2)$ invariance in the Higgs potential and maximal CP violation in Higgs-fermion interactions. Such a possibility, however, leads to a tightly constrained model (see Eq. (21) and appendix):

$$
\tan \beta \simeq 1, \quad m_{H^{0}} \simeq m_{H^{+}}
$$

If the discrete symmetries ${ }^{27}$ are exact symmetries of the lagrangian, the minimal model with $\mathrm{CP}$ violation is the Weinberg three Higgs doublet model ${ }^{28}$. If an 
$S U(2)_{L} \times S U(2)_{R}$ symmetry is imposed on the Higgs potential of this model, all the parameters are real. Thus, CP is conserved by the full lagrangian.

\section{Constraining CP violation from the experimental bounds on $T$}

Let us consider case I. Custodial $S U(2)$ symmetry requires the CP-odd state $A^{0}$ to be a mass eigenstate of mass equal to the charged Higgs mass (see appendix). Therefore, the custodial limit is given by

$$
\left\{\begin{array}{l}
\boldsymbol{O}_{31} \rightarrow 0, \\
\boldsymbol{O}_{32} \rightarrow 0, \\
\Delta m_{+3}^{2} \equiv m_{H^{+}}^{2}-m_{H_{3}}^{2} \rightarrow 0 .
\end{array}\right.
$$

The limit $\boldsymbol{O}_{31}, \boldsymbol{O}_{32} \rightarrow 0$ corresponds to $H_{3} \rightarrow A^{0}$, i.e., CP-conserving limit. In the limit (26), the contribution to $T$ (including only terms with quadratic dependence on the Higgs mass) can be written as

$$
\begin{aligned}
\alpha T & =\frac{g^{2}}{64 \pi^{2} m_{W}^{2}} \sum_{\substack{i, j=1 \\
i \neq j}}^{2} \boldsymbol{O}_{i 1}^{2}\left[\frac{1}{2}+\frac{m_{H_{j}}^{2}}{m_{H_{j}}^{2}-m_{H^{+}}^{2}}+\left(\frac{m_{H_{j}}^{2}}{m_{H_{j}}^{2}-m_{H^{+}}^{2}}\right)^{2} \ln \frac{m_{H^{+}}^{2}}{m_{H_{j}}^{2}}\right] \Delta m_{+3}^{2} \\
& +\frac{g^{2}}{64 \pi^{2} m_{W}^{2}}\left[F\left(m_{H_{1}}^{2}, m_{H^{+}}^{2}\right)+F\left(m_{H_{2}}^{2}, m_{H^{+}}^{2}\right)-F\left(m_{H_{1}}^{2}, m_{H_{2}}^{2}\right)\right] \boldsymbol{O}_{31}^{2} .
\end{aligned}
$$

Note that when $\Delta m_{+3}^{2}=0$ (custodial limit of a CP-conserving THDM ${ }^{14,29}$ ), there are still terms that grow quadratically with the heavy Higgs masses. Explicitly, we have for $m_{H^{+}}=m_{H_{3}} \gg m_{H_{1}}, m_{H_{2}}$,

$$
\alpha T \simeq \frac{g^{2} m_{H^{+}}^{2}}{64 \pi^{2} m_{W}^{2}} \boldsymbol{O}_{31}^{2}
$$

The experimental bounds on $T$ can be used to constrain $\boldsymbol{O}_{31}$ and limit the magnitude of CP violation in the Higgs-gauge sector. Notice that in fact only two $\left(\Delta m_{+3}^{2}\right.$ 
and $\boldsymbol{O}_{31}$ ) of the three custodial-breaking parameters in (26) can be constrained (see Eq. (27)). Nevertheless, it seems unnatural for the parameter $\boldsymbol{O}_{32}$ to be large while $\Delta m_{+3}^{2}$ and $\boldsymbol{O}_{31}$ are suppressed. Thus one expects that in constraining $\boldsymbol{O}_{31}$ one is actually constraining $\mathrm{CP}$ violation in the full Higgs sector.

To get an idea of the magnitude of such bounds, we parameterize the custodial limit (26) in terms of only one parameter, $\boldsymbol{O}_{31}$. In a first case (Fig. 2a), we consider:

$$
\begin{aligned}
\boldsymbol{O}_{32} & =\boldsymbol{O}_{31}, \\
m_{H_{3}} & =\left(1+\frac{1}{2} \frac{\boldsymbol{O}_{31}}{\boldsymbol{O}_{31}^{\text {max }}}\right) m_{H^{+}},
\end{aligned}
$$

where $\boldsymbol{O}_{31}^{\max } \equiv \sqrt{3} / 3$ is the value of $\boldsymbol{O}_{31}$ for maximal CP violation ${ }^{7}$. In a second case (Fig. 2b) we again consider, $\boldsymbol{O}_{32}=\boldsymbol{O}_{31}$ and in addition saturate the third limit in (26) $\left(m_{H^{+}}=m_{H_{3}}\right)$. In both cases we also assume $\boldsymbol{O}_{21} \simeq \boldsymbol{O}_{11}$ and $m_{H_{1}}=$ $\frac{1}{2} m_{H_{2}}=\frac{3}{2} m_{H^{+}}$.

When we study Fig. 2 we see that unfortunately the actual constraints are very sensitive to the different parameters of the model. The results are also very sensitive to how the custodial limit is taken. If we fix the ratio $\Delta m_{+3}^{2} / O_{31}$ in the custodial limit (26), the dependence of $T$ on $\boldsymbol{O}_{31}$ is linear and then the bounds are tight (Fig. 2a). However, if $\Delta m_{+3}^{2} / O_{31}$ goes to zero in the custodial limit (26), then $T$ depends quadratically on $\boldsymbol{O}_{31}$ and the bounds are less restrictive (Fig. 2b).

\section{Conclusions}

We have shown that in a THDM with maximal $\mathrm{CP}$ violation in the Higgs sector, there are large contributions to $\Delta \rho$ coming from the scalar sector. We have seen that this is due to the fact that any term in the Higgs potential that breaks $\mathrm{CP}$ also breaks the custodial $S U(2)$ symmetry and, therefore, contributes to $\Delta \rho$ at 
one-loop. This is true in the Higgs-gauge sector and can be generalized to models with a more extended Higgs sector.

We have used the experimental bounds on the parameter $T$ to constrain $\mathrm{CP}$ violation in the THDM Higgs sector. These constraints (Fig. 2) are found to be very dependent on the parameters of the model. At present, they are the only bounds

on CP nonconservation in the Higgs sector. When the limits on $T$ improve $^{23}$ $(\Delta T= \pm 0.1)$, the region of parameter space in the Higgs potential that allows for $\mathrm{CP}$ violation will be further constrained.

It is important to remark that when the Higgs masses are large the constraints on $\mathrm{CP}$ nonconservation are stronger. Note that this is opposite to the case where $\mathrm{CP}$ nonconservation is constrained using the electric dipole moment.

When Higgs-fermion interactions are considered, we have shown that it is possible to find a definition of the custodial symmetry which allows for CP violation. Such a possibility leads to other interesting constraints (Eq. (25)).

\section{Acknowledgements}

We gratefully acknowledge conversations with Jack Gunion, Howard Haber and Michael Peskin. We would like to thank Cliff Burgess and Dave Robertson for a critical reading of the manuscript. The work of A.P. was supported by a fellowship of the MEC (Spain). 


\section{APPENDIX}

In this appendix we derive the restrictions that the $S U(2)_{L} \times S U(2)_{R}$ symmetry places on the most general THDM potential of Eq. (2).

For case I, the most general $S U(2)_{L} \times S U(2)_{R}$ symmetric potential is given by

$$
\begin{aligned}
V\left(M_{1}, M_{2}\right) & =\frac{1}{2} m_{1}^{2} \operatorname{Tr}\left\{M_{1}^{\dagger} M_{1}\right\}+\frac{1}{2} m_{2}^{2} \operatorname{Tr}\left\{M_{2}^{\dagger} M_{2}\right\}-m_{12}^{2} \operatorname{Tr}\left\{M_{1}^{\dagger} M_{2}\right\} \\
& +\frac{1}{4} \lambda_{1} \operatorname{Tr}^{2}\left\{M_{1}^{\dagger} M_{1}\right\}+\frac{1}{4} \lambda_{2} \operatorname{Tr}^{2}\left\{M_{2}^{\dagger} M_{2}\right\} \\
& +\frac{1}{4} \lambda_{3} \operatorname{Tr}\left\{M_{1}^{\dagger} M_{1}\right\} \operatorname{Tr}\left\{M_{2}^{\dagger} M_{2}\right\}+\frac{1}{2} \lambda \operatorname{Tr}^{2}\left\{M_{1}^{\dagger} M_{2}\right\} \\
& +\frac{1}{4} \operatorname{Tr}\left\{M_{1}^{\dagger} M_{2}\right\}\left[\lambda_{6} \operatorname{Tr}\left\{M_{1}^{\dagger} M_{1}\right\}+\lambda_{7} \operatorname{Tr}\left\{M_{2}^{\dagger} M_{2}\right\}\right] .
\end{aligned}
$$

Using Eq. (17), it is easy to see that the above potential corresponds to Eq. (2) with

$$
m_{12}^{2}, \lambda_{6}, \lambda_{7} \in \mathbf{R}, \lambda=\lambda_{4}=\lambda_{5} \in \mathbf{R}
$$

Thus, all parameters of the Higgs sector are real (see Eq. (19) and Eq. (A.2)), and $\mathrm{CP}$ is conserved. Explicitly, we find that $A^{0}$ is a mass eigenstate with $m_{A^{0}}=$

$m_{H^{+}}$. As we expected, Eq. (11) holds and, then the contribution to $T$ that grows quadratically with the Higgs masses vanishes.

For case II, the most general potential that is invariant under $S U(2)_{L} \times S U(2)_{R}$ is given by

$$
\begin{aligned}
V\left(M_{21}\right) & =m^{2} \operatorname{Tr}\left\{M_{21}^{\dagger} M_{21}\right\}-\left(m_{12}^{2} \operatorname{det} M_{21}^{\dagger}+\text { h.c. }\right) \\
& +\lambda \operatorname{Tr}^{2}\left\{M_{21}^{\dagger} M_{21}\right\}+\lambda_{4} \operatorname{det}\left\{M_{21}^{\dagger} M_{21}\right\}+\frac{1}{2}\left[\lambda_{5} \operatorname{det}\left\{M_{21}^{\dagger}\right\}^{2}+\text { h.c. }\right] \\
& +\frac{1}{2}\left[\lambda^{\prime} \operatorname{det} M_{21}^{\dagger} \operatorname{Tr}\left\{M_{21}^{\dagger} M_{21}\right\}+\text { h.c. }\right] .
\end{aligned}
$$

This potential corresponds to Eq. (2) with

$$
m^{2}=m_{1}^{2}=m_{2}^{2} \quad, \quad \lambda=\lambda_{1}=\lambda_{2}=\frac{1}{2} \lambda_{3} \quad, \quad \lambda^{\prime}=\lambda_{6}=\lambda_{7} .
$$


It seems that in this case we can have explicit and spontaneous CP violation, because the scalar self-couplings and the VEVs can be complex. Nevertheless, using Eqs. (21) and (A.4) in Eq. (2), one can see that $H^{0}$ is a mass eigenstate (with $m_{H^{0}}=m_{H^{+}}$). Therefore, considering only the Higgs-gauge sector, we can define ${ }^{7} H^{0}$ to be CP-odd and the other neutral Higgs to be CP-even. 


\section{REFERENCES}

1. M. Kobayashi and M. Maskawa, Prog. Theor. Phys. 49 (1973) 652.

2. S. Weinberg, Phys. Rev. D42 (1990) 860.

3. S. Weinberg, Phys. Rev. Lett. 63 (1989) 2333.

4. J.F. Gunion, D. Wyler, Phys. Lett. B248 (1990) 170.

5. S.M. Barr and A. Zee, Phys. Rev. Lett. 65 (1990) 61; J.F. Gunion and R. Vega, Phys. Lett. B251 (1990) 157; R.G. Leigh et al., Nucl. Phys. B352 (1991) 45; D. Chang, W.-Y. Keung and T.C. Yuan, Phys. Rev. D43 (1991) 14

6. B. Grzadkowski and J.F. Gunion, Phys. Lett. B287 (1992) 237; C. Schmidt and M. Peskin, Phys. Rev. Lett. 69 (1992) 410; R. Cruz, B. Grzadkowski and J.F. Gunion, Phys. Lett. B289 (1992) 440; D. Atwood, G. Eilam and A. Soni, Phys. Rev. Lett. 70 (1993) 1364 ; preprint SLAC-PUB-6083.

7. A. Méndez and A. Pomarol, Phys. Lett. B272 (1991) 313.

8. B. Grzadkowski and J.F. Gunion, Phys. Lett. B294 (1992) 361.

9. A. Pilaftsis and M. Nowakowski, preprint MZ-TH-92-56 (1992); A. Soni and R.M. Xu, preprint BNL-48160 (1992); D. Chang, W.-Y. Keung and I. Phillips, preprint CERN-TH-6814-93 (1993); X.-G. He, J.P. Ma and B. McKellar, preprint UM-P-93-11 (1993).

10. P. Sikivie et al., Nucl. Phys. B173 (1980) 189.

11. G.C. Branco, J.-M. Gerard and W. Grimus, Phys. Lett. B136 (1984) 383.

12. T.D. Lee, Phys. Rev. D8 (1973) 1226.

13. D. Toussaint, Phys. Rev. D18 (1978) 1646. 
14. R.S. Lytel, Phys. Rev. D22 (1980) 505.

15. J.-M. Frère and J.A.M. Vermaseren, Z. Phys. C19 (1983) 63.

16. S. Bertolini, Nucl. Phys. B272 (1986) 77.

17. W. Hollik, Z. Phys. C32 (1986) 291.

18. C.D. Froggatt, I.G. Knowles and R.G. Moorhouse, Phys. Rev. D45 (1992) 2471; Nucl. Phys. B386 (1992) 63.

19. M.E. Peskin and T. Takeuchi, Phys. Rev. Lett. 65 (1990) 964.

20. F. Antonelli, M. Consoli, J. Corbo, Phys. Lett. B91 (1980) 90; M. Veltman, Phys. Lett. B91 (1980) 95.

21. L. Lavoura, Int. J. Mod. Phys. A8 (1993) 375.

22. D.C. Kennedy and P. Langacker, Phys. Rev. Lett. 65 (1990) 2967; Phys. Rev. D44 (1991) 1591.

23. A. Blondel, F.M. Renard and C. Verzegnassi, Phys. Lett. B269 (1991) 419.

24. J.F. Gunion, R. Vega and J. Wudka, Phys. Rev. D43 (1991) 2322.

25. E. Ma and D. Ng, preprint UCRHEP-T103 (1993).

26. H.E. Haber and A. Pomarol, Phys. Lett. B302 (1993) 435.

27. S. Glashow and S. Weinberg, Phys. Rev. D15 (1977) 1958.

28. S. Weinberg, Phys. Rev. Lett. 37 (1976) 657.

29. N.G. Deshpande and E. Ma, Phys. Rev. D18 (1978) 2574. 


\section{FIGURE CAPTIONS}

1) Extra contribution to $T$ from a THDM with maximal $\mathrm{CP}$ violation $\left(\boldsymbol{O}_{i 1}=\right.$ $\frac{\sqrt{3}}{3}$ ). The experimental allowed region corresponds to the region inside the dotted lines.

2) Extra contribution to $T$ from a THDM where CP violation is parametrized by $\boldsymbol{O}_{31}\left(\boldsymbol{O}_{31}^{\max }=\sqrt{3} / 3\right)$. The experimental allowed region corresponds to the region inside the dotted lines. 